\title{
ARGONAUTE9-dependent silencing of transposable elements in pericentromeric regions of Arabidopsis
}

\author{
Noé Durán-Figueroa and Jean-Philippe Vielle-Calzada* \\ Grupo de Desarrollo Reproductivo y Apomixis; Laboratorio Nacional de Genómica para la Biodiversidad y Departamento de Ingeniería Genética de Plantas; \\ Cinvestav Irapuato, México
}

Key words: gametogenesis, ARGONAUTE9, centromere, transposons, Arabidopsis

Submitted: 09/05/10

Accepted: 09/06/10

Previously published online:

www.landesbioscience.com/journals/psb/ article/ 13548

DOI: $10.4161 /$ psb.5.11.13548

*Correspondence to: Jean-Philippe Vielle-Calzada; Email: vielle@ira.cinvestav.mx

Addendum to: Olmedo-Monfil V, Durán-Figueroa N, Arteaga-Vázquez M, Demesa-Arévalo E, Autran D, Grimanelli D, et al. Control of female gamete formation by a small RNA pathway in Arabidopsis. Nature 2010; 464:628-32; PMID: 20208518; DOI: 10.1038/nature08828.
$\mathrm{R}$ ecent evidence indicates that the establishment of the haploid phase of the plant life cycle requires epigenetic mechanisms that control reproductive cell fate. We previously showed that in Arabidopsis thaliana (Arabidopsis) mutations in ARGONAUTE9 (AGO9) result in defective cell specification during megasporogenesis. AGO9 preferentially interacts with 24 nucleotide (nt) small RNAs (sRNAs) derived from transposable elements (TEs), and its sporophytic activity is required to silence TEs in the female gametophyte. Here we show that AGO9 can bind in vitro to $24 \mathrm{nt}$ sRNAs corresponding to Athila retrotransposons expressed in the ovule prior to pollination. We also show that AGO9 is necessary to inactivate a significant proportion of long terminal repeat retrotransposons (LTRs) in the ovule, and that its predominant TE targets are located in the pericentromeric regions of all five chromosomes, suggesting a link between the AGO9-dependent sRNA pathway and heterochromatin formation. Our extended results point towards the existence of a tissue-specific mechanism of sRNA-dependent TE silencing in the ovule.

The life cycle of flowering plants alternates between diploid (sporophytic) and haploid (gametophytic) generations (or phases) occurring in specialized reproductive organs. In most flowering plants, as in the young ovule of Arabidopsis, a single sub-epidermal cell differentiates into the megaspore mother cell (MMC) before entering meiosis and giving rise to a single viable haploid product, the functional megaspore, that undergoes three rounds of nuclear haploid divisions before differentiating into the female gametophyte. The establishment of the gametophytic phase presents an opportunity for natural selection to act on the haploid plant genome as an evolutionary driving force that could be at the origin of epigenetic mechanisms that ensure a tight regulation of reproductive development. ${ }^{1}$ Despite this early-acting selective pressure, there are numerous examples of developmental alternatives that suggest a flexible regulatory control of gamete formation. ${ }^{2}$

ARGONAUTE (AGO) proteins interact with a multitude of small non-coding RNAs (sncRNAs), such as microRNAs (miRNAs) and small interefering RNAs (siRNAs) that are crucial to regulate developmental mechanisms across eukaryotes. The epigenetic mechanisms that are controlled by the AGO-sRNA interactions are often dependent on the spatial and temporal control of their transcription. ${ }^{3} \mathrm{We}$ recently showed that an ARGONAUTE protein of Arabidopsis (AGO9) is an important regulator of early cell specification in the ovule. Heterozygous ago9/+ individuals show abnormal proliferation of gamete precursor cells that are able to divide and differentiate into functional female gametophytes. AGO9 is preferentially expressed in epidermal (L1) cells of the ovule primordium and is absent from the MMC, the meiotically derived megaspores, or the developing female gametophyte, indicating that it acts nonautonomously to restrict MMC identity to a single cell. We also identified a collection of AGO9-interacting sRNAs in which 


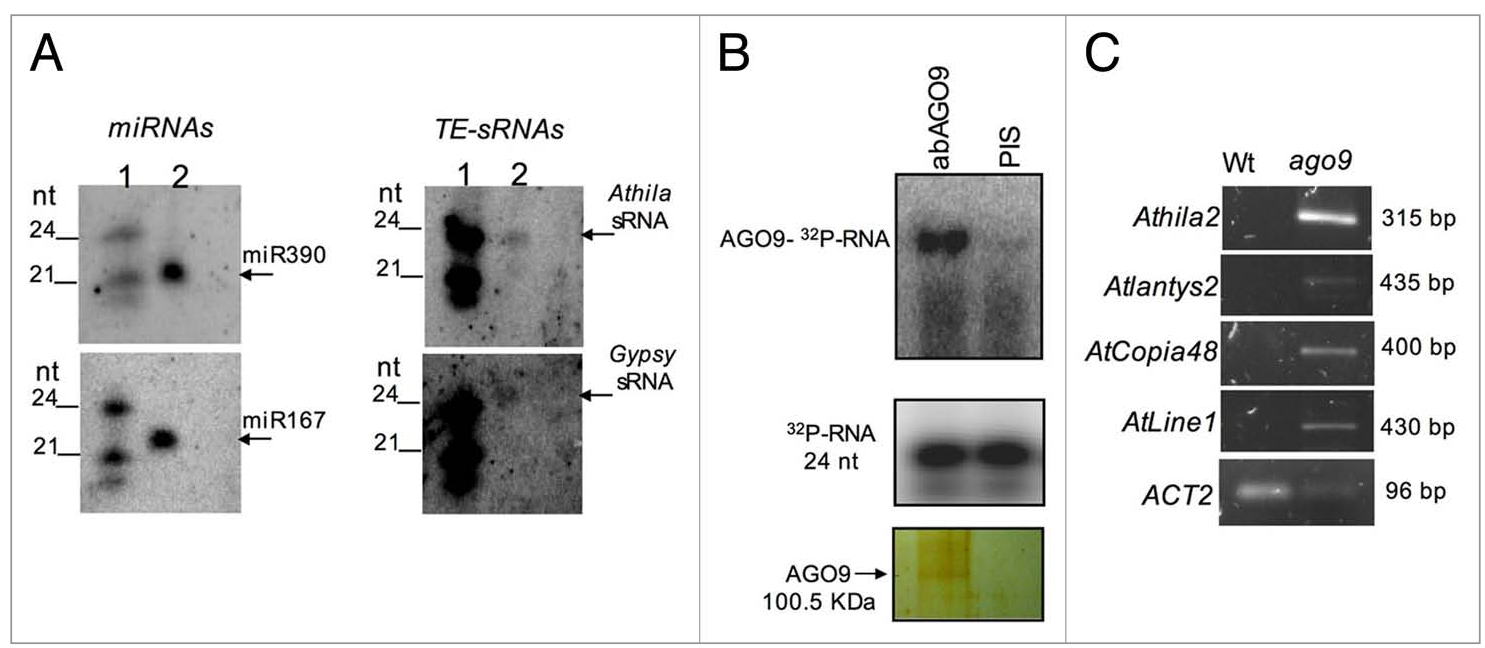

Figure 1. Expression of AGO9-interacting sRNAs and TEs in the ovule of Arabidopsis. (A) Selected AGO9 sRNA interactors are expressed in developing gynoecia prior to pollination. (1) molecular marker; (2) RNA from developing gynoecia. (B) Detection of a $24 \mathrm{nt}$ sRNA corresponding to an Athila TE in AGO9 (abAGO9) immunoprecipitate; immunopurification with pre-immune serum (PIS) was used as negative control. A radiolabeled $24 \mathrm{nt}$ RNA is shown to indicate that comparable amounts of protein were used for RNA preparation. (C) Multiple TE members of Athila2, Atlantys2, AtCopia48, and AtLINE are expressed in unpollinated ovules of homozygous ago9 individuals but not in wild-type unpollinated ovules. ACTIN2 (ACT2) RT-PCR amplification was used as a positive control.

a class that is $24 \mathrm{nt}$ in length and corresponds to TEs is largely predominant; however, some other members of this sRNA collection are 21 to $22 \mathrm{nt}$ in length and correspond to previously reported miRNAs or siRNAs. ${ }^{4}$

We conducted RNA blot analysis to confirm that selected AGO9-interacting sRNAs corresponding to LTRs of the Gypsy and Athila family, or to miRNAs such as miR167 and miR390, ${ }^{5,6}$ are expressed in female tissue of Arabidopsis. All four sRNAs tested were expressed in gynoecia prior to fertilization (Fig. 1A). To confirm that some of these previously identified sRNAs interact with AGO9, a 24 nt RNA oligonucleotide corresponding to an Athila retrotransposon was 5 '-end labeled and incubated with either immunopurified AGO9 protein, or the pre-immune AGO9 serum as a negative control. The reaction mixtures were subsequently irradiated with UV and resolved in SDS-PAGE. As shown in Figure 1B, AGO9 showed a strong binding affinity for the Athila-derived 24 nt sRNA, biochemically confirming that previously identified $24 \mathrm{nt}$ sRNAs derived from LTRs are interactors of AGO9 in the developing ovule.

We have shown that TEs of the Athila, Atlantys and LINE families that were tagged by enhancer trap lines are reactivated in the female gametophyte of heterozygous ago9/+ individuals. ${ }^{4}$ Additionally, it has been suggested that retrotransposons are transcribed in the vegetative cell of pollen to serve as a template for the biogenesis of sRNAs necessary to silence equivalent TEs in sperm cells. ${ }^{7}$ To determine if AGO9 is also required for inactivating a larger fraction of retrotrasposons of the Athila, Atlantys, AtCopia and AtLine family, we assess the overall expression of these families of TEs in the ovule by conducting reverse transcription PCR (RT-PCR) in mutant ago 9 and wild-type individuals, using primers that allow the amplification of multiple closely related member TEs in all four families. These same members were previously shown to be active in the vegetative nucleus of pollen but not in sperm cells. ${ }^{7}$ As shown in Figure 1C, all TE members tested are not normally active in wild-type ovules; however, in the absence of AGO9 activity, members in all four families are reactivated, indicating that $\mathrm{AGO} 9$ is of broad importance for ensuring TE silencing in the ovule. Because some AGO9 sRNA interactors are also expressed in developing endosperm following double fertilization, ${ }^{8}$ our results suggest that some of the sRNAs that are abundantly detected in the developing seed are of maternal origin and present in sporophytic cells before fertilization.
Large-scale transcriptional analysis has revealed that a multitude of polymeraseIV(polIV)-dependent sRNAs are expressed in the developing seed specifically from maternal chromosomes. Using bioinformatic tools, we identified the genomic location of all previously reported sRNA members of the AGO9 interacting collection and compare them to previously identified polI $V$-dependent sRNAs. Whereas most members do not match previously identified polI $V$-dependent sRNAs, 13 members correspond to locus 00687, a polIV-dependent region rich in maternal sRNAs abundantly present in the endosperm. Strikingly, the vast majority of TEs corresponding to AGO9 sRNA interactors map to centromeric and pericentromeric regions in all five Arabidopsis chromosomes (Fig. 2), suggesting a link between AGO9 function and heterochromatin formation.

Taken together, these results extend our previous findings by demonstrating that AGO9 plays a broad role in the inactivation of pericentromeric TEs in the ovule, particularly LTR-retrotransposons of the Gypsy and Athila families, a finding that is in agreement with previous evidence suggesting an important role for AGO proteins that interact with $24 \mathrm{nt} s \mathrm{RNAs}$ in silencing repetitive genomic regions involved in heterochromatin formation..$^{10,11}$ 


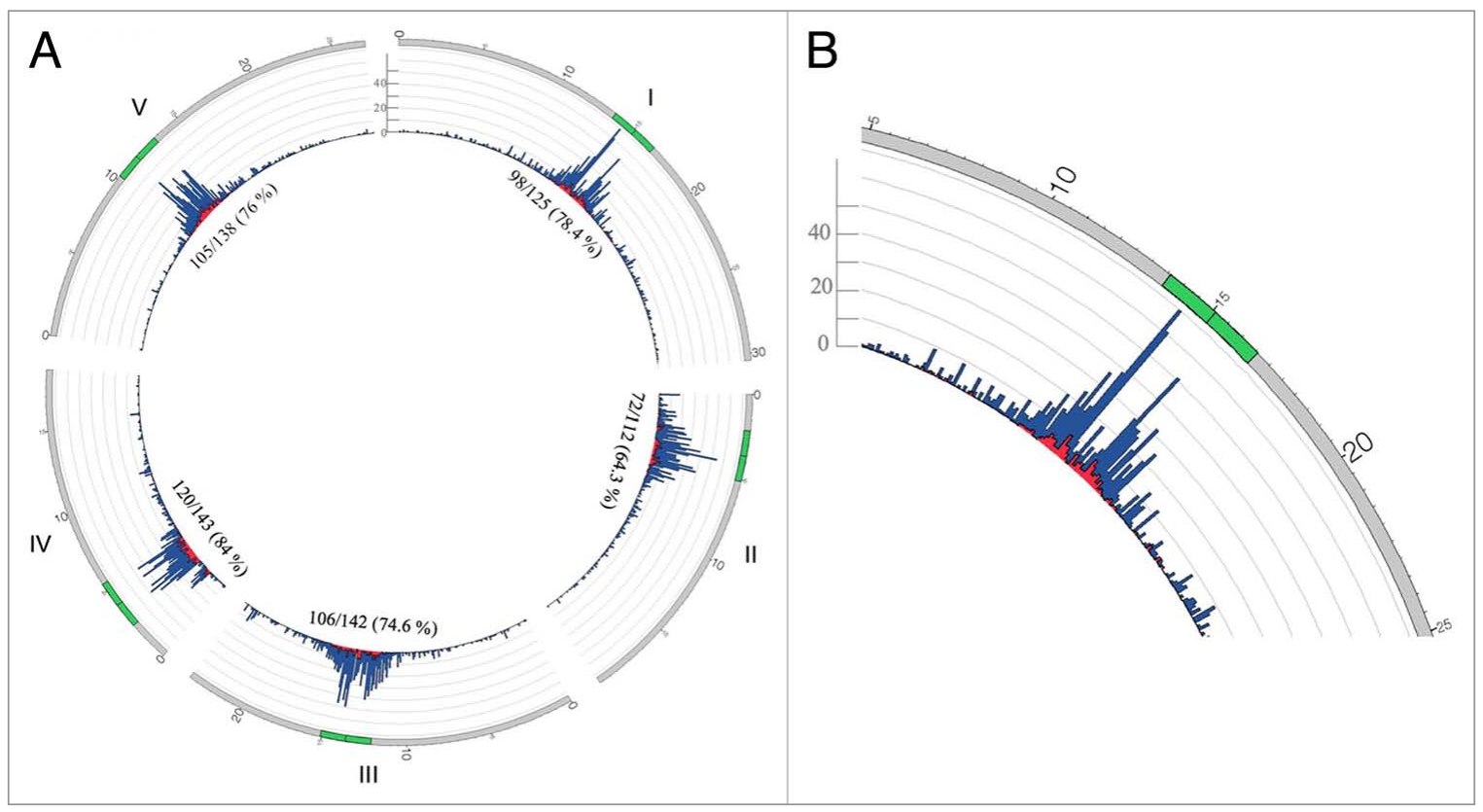

Figure 2. Genomic location of TEs corresponding to AGO9 sRNA interactors. (A) The vast majority of known AGO9 sRNA interactors corresponding to Athila or Gypsy TEs map to pericentromeric regions in all 5 Arabidopsis chromosomes (the length of the pericentromeric region is defined according to Hosouchi et al. ${ }^{16}$ ). Histograms show the total number of genomic Athila and Gypsy TEs (blue), and the total number of AGO9 interacting sRNAs that correspond to Athila and Gypsy TEs (red) located within arbitrary genomic segments of $100 \mathrm{~Kb}$, and this for all 5 chromosomes (Mb). The total number of AGO9 interacting sRNAs corresponding to Athila and Gypsy TEs over the total number of AGO9 interacting sRNAs identified in each chromosome is also indicated. (B) Detail of the pericentromeric region of Chromosome I.

We also confirmed that some of the $24 \mathrm{nt}$ AGO9 interactors are dependent on polIV for their transcription. Interestingly, some of them are expressed in the ovule prior to pollination, indicating that their detection in the developing endosperm could be the result of their movement from sporophytic maternal cells into the developing endosperm, rather than from their uniparental transcription following fertilization of the central cell. ${ }^{12}$ Our results also extend recent findings showing that 24 nt sRNAs can move from the vegetative cell cytoplasm to the sperm cells and silence TEs, a mechanism that is likely to be controlled by a similar silencing pathway to the one prevailing in the ovule. Although the molecular mechanisms that control this silencing route remain to be elucidated, it is clear that the functional characterization of the ARGONAUTE9-dependent pathway will open new possibilities to understand and manipulate the epigenetic control of gametogenesis in plants.

\section{Methods}

Plant material and growth conditions. Seeds from Arabidopsis thaliana ecotype
Columbia (Col-0) were surface-sterilized by washing three times with $100 \%$ ethanol and plated on Murashige and Skoog (MS) medium. MS plates containing seeds were placed in full darkness for three $\mathrm{d}$ at $4^{\circ} \mathrm{C}$, and subsequently germinated in a growth chamber at $22^{\circ} \mathrm{C}$ under a $16 \mathrm{~h}$ light $/ 8 \mathrm{~h}$ dark photoperiod, transferred to soil, and grown in the greenhouse under long day $16 \mathrm{~h}$ light/ 8 $\mathrm{h}$ dark controlled conditions.

Small RNA northern blot and UV-crosslinking assay. Northern blot analysis with enriched small RNAs was performed as described, ${ }^{13}$ with some modifications. The sRNA fraction was purified from more than 2,000 gynoecia using the mirVana miRNA isolation kit (Ambion) in accordance with manufacturer's instructions. The sRNA fraction was electrophoretically separated on a 12.5\% 19:1 acrylamide:bisacrylamide gel with $1 \mathrm{x}$ TBE-7 M Urea and visualized by SYBR-gold (Invitrogen) staining, before being transferred to Hybond NX membranes (GE/Amersham) in 1x TBE at 10 $\mathrm{V}$ for $1 \mathrm{~h}$. Membranes were crosslinked with 1-Ethyl-3-(3 dimethylaminopropyl) carbodiimide (EDC, Sigma) before pre-hybridization in northernMax buffer (Ambion) at $55^{\circ} \mathrm{C}$ for $1 \mathrm{~h}$. Oligonucleotides $(20 \mathrm{pmol})$ were labelled with $\left[\gamma^{-32} \mathrm{P}\right]$ ATP and T4 polynucleotide kinase (Invitrogen). Hybridization of labeled oligonucleotides occurred overnight in northernMax buffer at $55^{\circ} \mathrm{C}$. Membranes were washed twice in $2 \mathrm{x}$ SSC, $0.1 \%$ SDS at $55^{\circ} \mathrm{C}$, and exposed to phosphor-storage screens. Oligonucleotide sequences are 5'-AGA ACA AGG ATG TTG AAG AAG GCT-3' for Athila sRNA, 5'-AGT GAC TAG ACA AGG GAA ACG ACT-3' for Gypsy, 5'-GGC GCT ATC CCT CCT GAG CTT-3' for miR390 and 5'-TAG ATC ATG CTG GCA GCT TCA-3' for miR167. The UV-crosslinking assay was performed as described Mi et al. ${ }^{14}$ For this assay we use an RNA oligo provided by Exiqon (5'-AGA ACA AGG AUG UUG AAG AAG GCU-3').

RT-PCR. Total RNA was extracted from unpollinated mature ovules prior using TRIzol (Invitrogen), and 1 ug was used to synthesize first-strand cDNA by using 20mer-oligodTs (Sigma), 0.25 $\mathrm{mM}$ dNTPs and SuperScript III Reverse Transcriptase (Invitrogen), and incubating at $42^{\circ} \mathrm{C}$ for $2 \mathrm{~h}$. Primer sequence and 
PCR conditions were according to Slotkin et al. ${ }^{7}$ ACTIN2 mRNA was used as a control.

Bioinformatic analysis. Annotation of sRNAs was performed using genomic information from TAIR9 and the location of small RNA in all five chromosomes of Arabidopsis was generated using Perl Circos $^{15}$ and Chromosome Map Tool (www. arabidopsis.org/).

\section{Acknowledgements}

We thank Jaime Mendiola for assistance with sample collection, Cesar Alvarez for help with bioinformatic analysis, and all members of the Apolab for suggestions. N.D.F. was supported by a Ph.D. scholarship from Consejo Nacional de Ciencia y Tecnología (CONACyT). The research is funded by CONACyT and the Howard Hughes Medical Institute International Scholar program.

\section{References}

1. Walbot V, Evans MM. Unique features of the plant life cycle and their consequences. Nat Rev Genet 2003; 4:369-79.

2. Yang WC, Shi DQ, Chen YH. Female gametophyte development in flowering plants. Annu Rev Plant Biol 2010; 61:89-108.

3. Havecker ER, Wallbridge LM, Hardcastle TJ, Bush MS, Kelly KA, Dunn RM, et al. The Arabidopsis RNA-directed DNA methylation argonautes func tionally diverge based on their expression and interaction with target loci. Plant Cell 2010; 22:321-34.

4. Olmedo-Monfil V, Duran-Figueroa N, ArteagaVazquez M, Demesa-Arevalo E, Autran D, Grimanelli D, et al. Control of female gamete formation by a small RNA pathway in Arabidopsis. Nature 2010; 464:628-32.

5. Wu MF, Tian Q, Reed JW. Arabidopsis microRNA167 controls patterns of ARF6 and ARF8 expression and regulates both female and male reproduction. Development 2006; 133:4211-8.

6. Vazquez F, Legrand S, Windels D. The biosynthetic pathways and biological scopes of plant small RNAs. Trends Plant Sci 2010.

7. Slotkin RK, Vaughn M, Borges F, Tanurdzic M, Becker JD, Feijo JA, et al. Epigenetic reprogramming and small RNA silencing of transposable elements in pollen. Cell 2009; 136:461-72.

8. Mosher RA, Melnyk CW, Kelly KA, Dunn RM, Studholme DJ, Baulcombe DC. Uniparental expression of PolIV-dependent siRNAs in developing endosperm of Arabidopsis. Nature 2009; 460:283-6.
9. Mosher RA, Schwach F, Studholme D, Baulcombe DC. PolIVb influences RNA-directed DNA methylation independently of its role in siRNA biogenesis. Proc Natl Acad Sci USA 2008; 105:3145-50.

10. Henderson IR, Jacobsen SE. Epigenetic inheritance in plants. Nature 2007; 447:418-24.

11. Teixeira FK, Colot V. Repeat elements and the Arabidopsis DNA methylation landscape. Heredity 2010; 105:14-23

12. Mosher RA, Melnyk CW. siRNAs and DNA methylation: seedy epigenetics. Trends Plant Sci 2010; 15:204-10.

13. Pall GS, Hamilton AJ. Improved northern blot method for enhanced detection of small RNA. Nat Protoc 2008; 3:1077-84.

14. Mi S, Cai T, Hu Y, Chen Y, Hodges E, Ni F, et al. Sorting of small RNAs into Arabidopsis argonaute complexes is directed by the 5' terminal nucleotide. Cell 2008; 133:116-27.

15. Krzywinski M, Schein J, Birol I, Connors J, Gascoyne $\mathrm{R}$, Horsman D, et al. Circos: an information aesthetic for comparative genomics. Genome Res 2009; 19:1639-45.

16. Hosouchi T, Kumekawa N, Tsuruoka H, Kotani H. Physical map-based sizes of the centromeric regions of Arabidopsis thaliana chromosomes 1-3. DNA Res 2002; 9:117-21. 